\title{
Metastasis via Peritumoral Lymphatic Dilation in Oral Squamous Cell Carcinoma
}

\author{
Han-Seok Kim, Young-Wook Park \\ Department of Oral and Maxillofacial Surgery, College of Dentistry, Gangneung-Wonju National University
}

\begin{abstract}
Purpose: Nodal metastasis is the main prognostic factor in the patients with oral squamous cell carcinoma (OSCC). We investigated the association between tumor-associated lymphatics and OSCC characteristics.

Methods: Thirty-four specimens were used for the immunohistochemical staining with the antibody for vascular endothelial growth factor (VEGF)-C, VEGF-D, VEGF receptor (VEGFR)-3, phosphorylated VEGFR-3, D2-40, and matrix metallproteinases (MMPs). We observed the distribution of the lymphangiogenic factors and quantified the degree of expression. We determined lymphatic vessel density (LVD) and lymphatic vessel dilatation with D2-40 immunostaining. We assessed the association of LVD or lymphatic vessel dilatation with tumor progression or tumor differentiation.

Results: OSCC cells expressed lymphangiogenic ligands. Lymphangiogenic receptor, VEGFR-3, was expressed and activated in some tumor cells as well as in tumor-associated endothelial cells. LVD was not associated with tumor size or nodal status, but lymphatic vessel dilatation was higher in tumors with nodal metastasis, and also higher in poorly differentiated tumors. In stromal area of OSCC, MMP-1 and MMP-10 were up-regulated and the basement membrane of tumor-associated endothelial cells was destroyed by these collagenases.

Conclusion: In the primary tumors with nodal metastasis, especially in poorly differentiated OSCC, tumor cells invaded the dilated lymphatic vessels via ruptured sites. MMP-1 and MMP-10 are important in the lysis of the glycocalyx inside the tumor-associated lymphatic endothelial cells.
\end{abstract}

Key words: Oral squamous cell carcinoma, Lymphangiogenic factor, Nodal metastasis, Lymphatic vessel density, Lymphatic vessel dilation

\section{Introduction}

Nodal metastasis is a critical step in the progression of malignant tumors and is one of the most important prognostic factors in many types of epithelial solid tumors. In oral squamous cell carcinoma as well as head and neck carcinoma, tumor metastasis occurs mainly via cervical lymph nodes, and early cervical nodal involvement of the tumor results in poor survival[1].

Lymphangiogenesis, i.e., the formation of new lymphatic vessels, has a major effect on nodal metastasis of malignant tumors, regulated by the expression of lymphangiogenic factors[2,3]. The exact molecular mechanism of lymphangiogenesis and the biology of tumor-associated

RECEIVED May 7, 2014, REVISED May 7, 2014, ACCEPTED May 26, 2014

Correspondence to Young-Wook Park

Department of Oral and Maxillofacial Surgery, College of Dentistry, Gangneung-Wonju National University

7 Jukheon-gil, Gangneung 210-702, Korea

Tel: 82-33-640-3183, Fax: 82-33-640-3103, E-mail: ywpark@gwnu.ac.kr

Copyright (C) 2014 by The Korean Association of Maxillofacial Plastic and Reconstructive Surgeons. All rights reserved.

(c) This is an open access article distributed under the terms of the Creative Commons Attribution Non-Commercial License (http://creativecommons. org/licenses/ by-nc/3.0) which permits unrestricted non-commercial use, distribution, and reproduction in any medium, provided the original work is properly cited. 
lymphatic endothelial cells however, is unclear. Specific markers for lymphatic endothelium (Prox1[4], LYVE-1[5], podoplanin[6], and vascular endothelial growth factor receptor (VEGFR)-3[7]) have recently been discovered, leading to increased research interest in tumor lymphatics.

Among several lymphangiogenic factors, the vascular endothelial growth factor (VEGF)-C, D/VEGFR-3 signaling pathway is the most powerful stimulant for the genesis of tumor-associated lymphatics[8]. Research shows an association of VEGF-C/VEGFR-3 axis with lymphatic spread of tumor cells and with clinical outcomes in solid tumors including oral squamous cell carcinoma (OSCC)[9-11].

The main hypothesis of this study is that lymphangiogenic factor levels are higher in the primary tumor with nodal metastasis than in the primary tumor without nodal metastasis in OSCC. Thus we assessed the association of tumor-associated lymphatics with tumor proliferation or nodal metastasis or tumor differentiation.

\section{Materials and Methods}

\section{Patients and tumor specimens}

Thirty-four surgical specimens were collected from patients diagnosed and treated in the Department of Oral and Maxillofacial Surgery, Gangneung-Wonju National University Dental Hospital and Gangneung Asan Hospital between 2006 and 2010. The mean age of the 34 patients (23 males/11 females) was 63.5, ranging from 27 to 84 years. Clinicopathological data were collected (GNAH IRB No 2011-020). Clinical and basic tumor characteristics are shown in Table 1. Tumor grading was performed by an oral pathologist using World Health Organization (WHO) classification, and staging by UICC (Union Internationale Contre le Cancer, International Union against Cancer) classification.

Table 1. Patient and tumor profiles

\begin{tabular}{|c|c|c|c|c|c|c|c|}
\hline Patient No. & Age $(y r)$ & Sex & Location & Tumor size* & Nodal metastasis* & Distant metastasis* & TNM stage* \\
\hline 1 & 50 & Male & Left buccal mucosa & T1 & No & M0 & I \\
\hline 2 & 48 & Male & Left mouth floor & T1 & No & M0 & 1 \\
\hline 3 & 27 & Male & Tongue & $\mathrm{T} 2$ & NO & MO & II \\
\hline 4 & 63 & Female & Right palate & $\mathrm{T} 2$ & No & M0 & II \\
\hline 5 & 49 & Female & Retromolar area & $\mathrm{T} 2$ & NO & MO & II \\
\hline 6 & 68 & Male & Right buccal mucosa & $\mathrm{T} 2$ & NO & MO & II \\
\hline 7 & 61 & Male & Right buccal mucosa & $\mathrm{T} 2$ & NO & M0 & II \\
\hline 8 & 64 & Male & Oral cavity & $\mathrm{T} 2$ & NO & MO & II \\
\hline 9 & 68 & Female & Left buccal mucosa & $\mathrm{T} 2$ & No & M0 & II \\
\hline 10 & 51 & Female & Tongue & $\mathrm{T} 2$ & No & MO & II \\
\hline 11 & 78 & Male & Left buccal mucosa & $\mathrm{T} 2$ & No & MO & II \\
\hline 12 & 70 & Male & Gingival mucosa & $\mathrm{T} 2$ & NO & MO & II \\
\hline 13 & 48 & Female & Tongue & T1 & NO & MO & I \\
\hline 14 & 66 & Male & Tongue & T2 & NO & MO & II \\
\hline 15 & 74 & Female & Left buccal mucosa & $\mathrm{T} 2$ & NO & MO & I \\
\hline 16 & 59 & Male & Oral cavity & $\mathrm{T} 2$ & N1 & MO & III \\
\hline 17 & 63 & Male & Mouth floor & $\mathrm{T} 4$ & $\mathrm{~N} 2$ & MO & IV \\
\hline 18 & 57 & Male & Tongue & $\mathrm{T} 4$ & NO & MO & IV \\
\hline 19 & 49 & Male & Mouth floor & T4 & NO & MO & IV \\
\hline 20 & 84 & Female & Left buccal mucosa & T3 & N2 & M0 & IV \\
\hline 21 & 65 & Female & Buccal mucosa & $\mathrm{T} 2$ & $\mathrm{~N} 2$ & MO & IV \\
\hline 22 & 67 & Female & Right palate & $\mathrm{T} 4$ & N2 & MO & IV \\
\hline 23 & 62 & Male & Left buccal mucosa & $\mathrm{T} 4$ & NO & MO & IV \\
\hline 24 & 53 & Male & Left mandible, gingiva & $\mathrm{T} 4$ & No & MO & IV \\
\hline 25 & 59 & Male & Right buccal area & $\mathrm{T} 4$ & N2 & MO & IV \\
\hline 26 & 61 & Male & Oral cavity & $\mathrm{T} 2$ & N1 & M1 & IV \\
\hline 27 & 72 & Male & Mouth floor & $\mathrm{T1}$ & N1 & MO & III \\
\hline 28 & 69 & Male & Tongue & $\mathrm{T} 2$ & $\mathrm{~N} 2$ & M1 & IV \\
\hline 29 & 57 & Female & Mouth floor & T3 & N2 & MO & IV \\
\hline 30 & 64 & Male & Soft palate & $\mathrm{T} 2$ & N1 & M1 & IV \\
\hline 31 & 74 & Male & Oral cavity & $\mathrm{T} 4$ & $\mathrm{~N} 2$ & MO & IV \\
\hline 32 & 62 & Male & Tongue & T3 & $\mathrm{N} 2$ & MO & IV \\
\hline 33 & 69 & Female & Tongue & T3 & $\mathrm{N} 2$ & MO & IV \\
\hline 34 & 50 & Male & Retromolar area & T3 & N2 & MO & IV \\
\hline
\end{tabular}

*Classification according to the TNM stage. 


\section{Antibodies}

Antibodies were purchased as follows: polyclonal antibodies specific for VEGF-C (dilution 1:100), VEGF-D (dilution 1:100; Abnova, Jhongli, Taiwan), VEGFR-3 (dilution 1:400, rabbit anti-human FLT-4; Spring Bioscience, Pleasanton, CA, USA), phosphorylated VEGFR-2/3 (dilution 1:500, pVEGFR-3; Calbiochem, Darmstadt, Germany), mouse monoclonal antibody specific for D2-40 (dilution 1:40; Abcam, Cambridge, MA, USA), matrix metalloproteinase (MMP)-1, MMP-2, MMP-9, and MMP-10 (dilution 1:100; Santa Cruz Biotechnology, Santa Cruz, CA, USA).

\section{Immunohistochemistry}

Immunohistochemical staining was performed on 5 $\mu \mathrm{m}$-thick sections. Paraffin sections were de-paraffinized in xylene, dehydrated with a graded series of alcohol, and rehydrated in phosphate buffered saline. For antigen retrieval, sections for VEGF-C and VEGFR-3 were microwaved in citrate buffer, and sections for VEGF-D and pVEGFR-3 were microwaved in EDTA buffer. Except for the staining with D2-40 and MMPs, immunohistochemical staining was performed in DakoCytomation Autostainer ${ }^{\mathrm{TM}}$ (Bond-Max; Leica Biosystems, Newcastle, UK) with envision+horseradish peroxidase diaminobenzidine.

For D2-40 staining, tissue sections treated with the primary antibodies were incubated overnight at 100\% humidity in temperature chambers at $4{ }^{\circ} \mathrm{C}$. After adequate binding of the primary antibodies, biotinylated link solution as a secondary antibody was applied to the tissue sections for 30 minutes. After washing to remove any unbound secondary antibodies, streptavidin peroxidase solution was applied for 30 minutes. For colorization, diaminobenzidine solution was applied for less than five minutes. Mayer's hematoxylin was used for counter-staining. After gradational hydration with ethyl alcohol and clearing with xylene, the tissue sections were fixed by paramount solutions. MMPs staining was performed as above.

\section{Quantitative immunohistochemical analysis}

Tissue-slides were observed with a microscope camera (Olympus DP70 digital microscope camera; Olympus Co., Tokyo, Japan). Images were taken from two representative areas per specimen under $\times 100$ magnification. The images were blindly assessed to prevent area bias. To quantify the expression of each protein, an image analysis program (SigmaScan Pro 5.0; SPSS Science, Chicago, IL, USA) was used. The expression level was measured by the numbers of pixels that appeared red by this program. The average percent of the ratio of red pixels to total pixels was used as a measure of protein expression for each specimen.

\section{Lymphatic vessel density and lymphatic vessel dilatation}

Lymphatic vessel density (LVD) and lymphatic vessel dilatation were determined with immunostaining with D2-40. To determine LVD, the sections were scanned by light microscopy at a low magnification $(\times 100)$. We identified the area of the greatest number of lymph vessels each in intratumoral and peritumoral area. LVD was then determined by counting immunostained vessels that have lumina at higher magnification $(\times 200)$. Counts were performed blindly by two investigators. The frequency of lymphatic vessel dilation was determined by an oral pathologist from the same fields.

\section{Statistics}

The association between expressions of the lymphangiogenic factors and tumor size (T1, T2 vs. T3, T4) or nodal status (lymph node- [L/N-] vs. L/N+) was measured by the non-parametric Mann-Whitney test. The association between expressions of the lymphangiogenic factors and grade of tumor was measured by the non-parametric Kruskal-Wallis test. The associations between LVD or lymphatic vessel dilatation with tumor size or nodal metastasis were measured by the non-parametric Mann-Whitney test. The association between LVD or lymphatic vessel dilatation with grade of tumor was measured by the non-parametric Kruskal-Wallis test. Null hypotheses of no difference were rejected if $P$-values were less than 0.05 . All statistical calculations used SPSS ver. 17.0.1 (SPSS Inc., Chicago, IL, USA).

\section{Results}

\section{Histopathology}

Classification by tumor size resulted in four OSCC as T1, 17 OsCC as T2, five OSCC as T3, and eight OSCC 
as T4. Nineteen patients presented with lymph node metastasis (L/N+ group); 15 patients did not (L/N- group). By histological grading, 14 tumors were well differentiated (well group), nine tumors were moderately differentiated (moderately group), and 11 tumors were poorly differentiated (poorly group).
2. Expression of lymphangiogenic factors in oral squamous cell carcinoma

VEGF-C was expressed weakly in OSCC tumor cells in eight of 34 patients. VEGF-D expression could be detected in most tumor cells i.e., 29 of 34 patients, tumor-associated endothelial cells, and stromal cells such as mononuclear
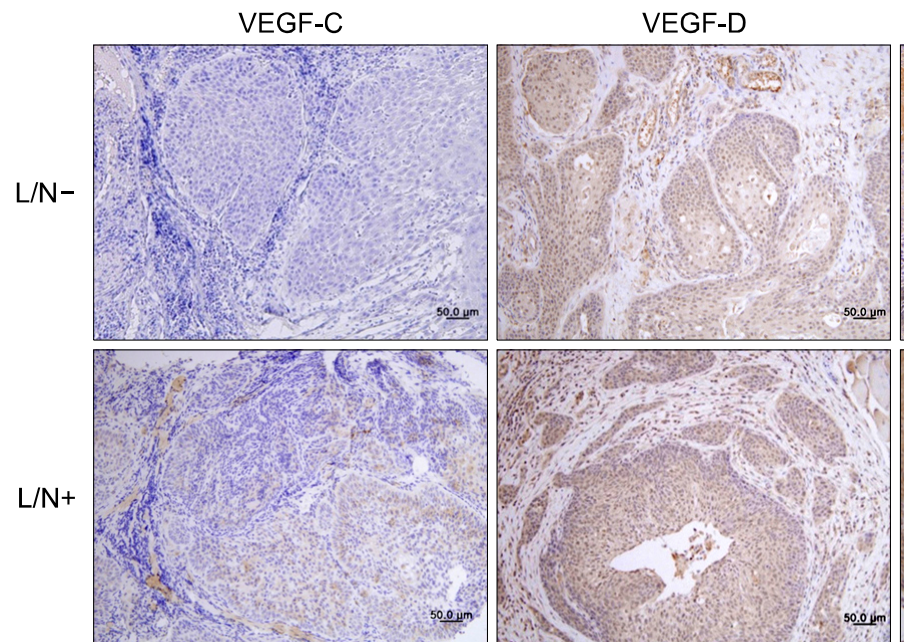
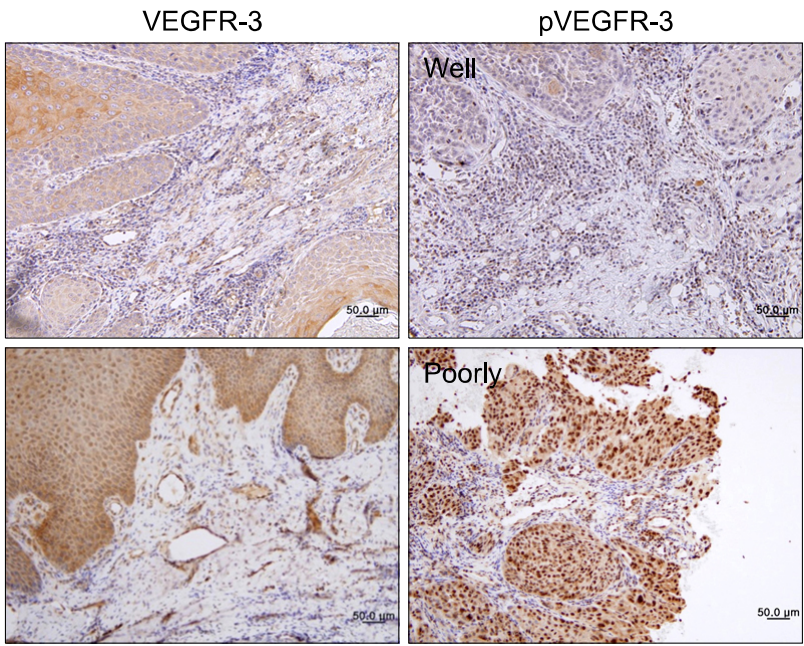

Fig. 1. Expression and distribution of lymphangiogenic factors in oral squamous cell carcinoma; VEGF-C is expressed weakly in some tumor cells. VEGF-D is expressed in most tumor cells, tumor-associated endothelial cells, and immune cells in stroma. VEGFR-3 is expressed strongly in some tumor cells, endothelial cells, and macrophages. Phosphorylated VEGFR-3 is strongly expressed in invading tumor cells in poorly differentiated tumors. Upper panel belongs to lymph node- $(\mathrm{L} / \mathrm{N}-)$ group and lower panel belongs to $\mathrm{L} / \mathrm{N}+\mathrm{group}$. The picture of pVEGFR-3 is the poorly group. Representative slides are shown $(\times 200)$ (counterstained by Mayer's hematoxyline).
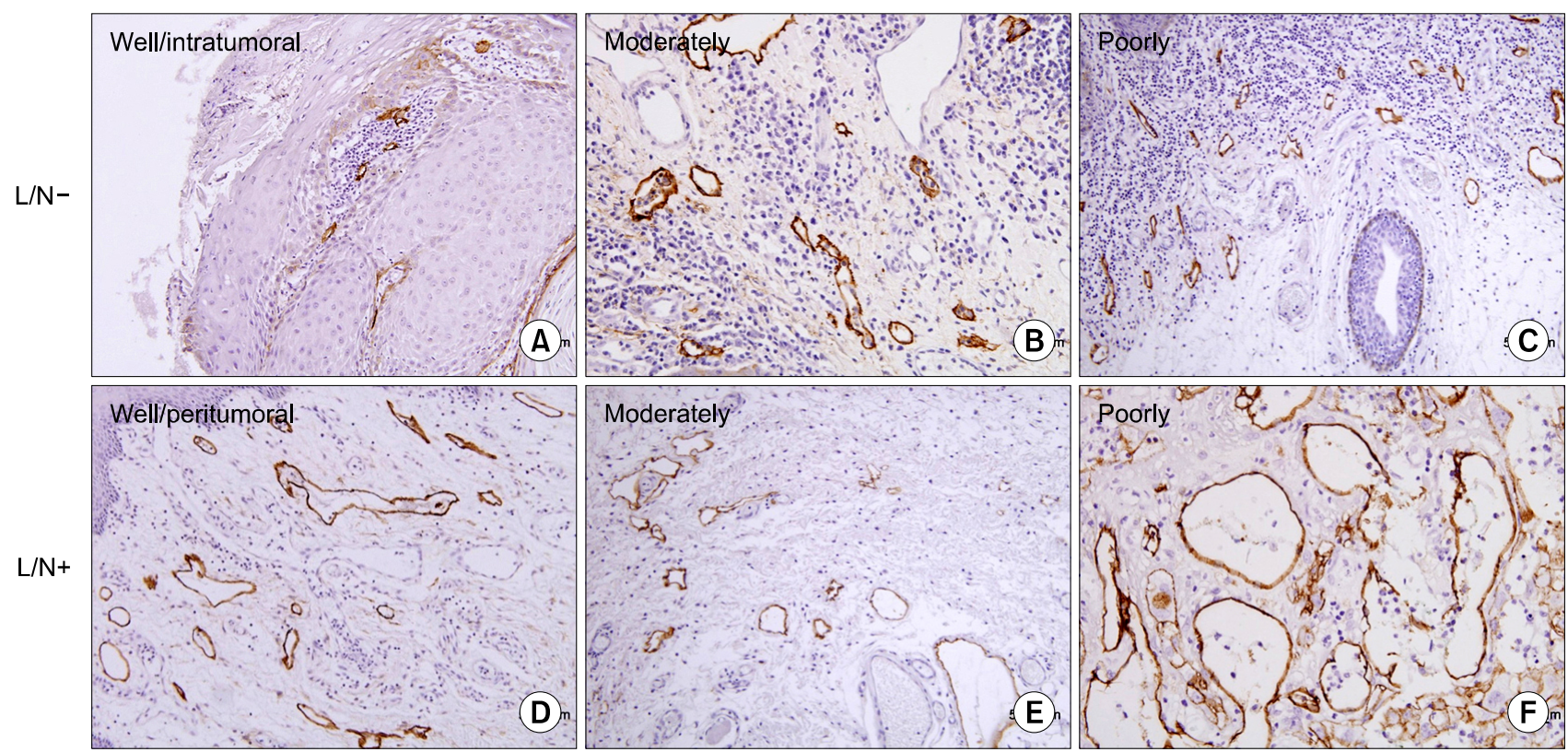

Fig. 2. Lymphatic vessel density (LVD) and lymphatic vessel dilatation; structures stained with D2-40 (brown) are compressed, apparently non-functional lymphatics (A: intratumoral LVD). Functional lymphatics are dilated and have larger surface area (D-F: peritumoral LVD), higher number in lymph node+ $(\mathrm{L} / \mathrm{N}+)$ group. We can see clearly the unstained microvessels $(A \sim D)$. Upper panel is $L / N-$ group and lower panel is L/N+ group. Representative slides are shown $(\mathrm{A} \sim \mathrm{F}: \times 200)$ (counterstained by Mayer's hematoxyline). 
cells and macrophages. However, the degree of VEGF-D expression on tumor cells was heterogenous. The degree of VEGF-C or VEGF-D expression was not related to the tumor size, nodal metastasis, or tumor grading.

VEGFR-3 expression in OSCC tumor tissue was found in some tumor cells, tumor-associated endothelial cells, and macrophages. VEGFR-3 was activated in invading tumor cells in primary tumor, endothelial cells, and some stromal cells of OSCC microenvironment (Fig. 1). However, the degree of VEGFR-3 or pVEGFR-3 expression was not related to the tumor size or nodal metastasis (data not shown). pVEGFR-3 expression was significantly higher in the poorly differentiated tumors (Table 2).

\section{Lymphatic vessel density and lymphatic vessel dilatation}

D2-40 was specifically expressed in the endothelial cells of tumor-associated lymphatic vessels. D2-40 positive lymphatic vessels were present in all OSCCs. The stained vessels were devoid of red blood cells, and D2-40 was restricted to the endothelium not only at the peritumoral lesion but also at the intratumoral lesion. Peritumoral LVD was higher than intratumoral LVD (Fig. 2). We could detect lymphatic vessel dilation and rupture in peritumoral lymphatics, but not in intratumoral lymphatics (Fig. 3).

Table 2. Expression of lymphangiogenic factors depending on tumor differentiation

\begin{tabular}{lccc}
\hline & $\begin{array}{c}\text { Well } \\
(n=14)\end{array}$ & $\begin{array}{c}\text { Moderately } \\
(n=9)\end{array}$ & $\begin{array}{c}\text { Poorly } \\
(n=11)\end{array}$ \\
\hline VEGF-C & $0.071 \pm 0.267$ & $0.25 \pm 0.50$ & $0.75 \pm 0.95$ \\
VEGF-D & $2.214 \pm 0.699$ & $2.25 \pm 0.95$ & $2.50 \pm 1.00$ \\
VEGFR-3 & $2.143 \pm 0.949$ & $1.25 \pm 0.50$ & $2.50 \pm 0.57$ \\
pVEGFR-2/3 & $1.571 \pm 1.158$ & $0.50 \pm 0.57$ & $2.75 \pm 0.50^{*}$ \\
\hline
\end{tabular}

Values are presented as mean \pm standard deviation. Well: well differentiated OSCC, Moderately: moderately differentiated OSCC, Poorly: poorly differentiated OSCC.

OSCC, oral squamous cell carcinoma.

${ }^{*} P<0.05$ compared with Moderately group (Kruskal-Wallis test).

Table 3. LVD depending on nodal status

\begin{tabular}{lccc}
\hline & Group $1(n=19)$ & Group $2(n=15)$ & $P$-value \\
\hline Intratumoral LVD & $6.04 \pm 4.57$ & $6.88 \pm 3.61$ & 0.136 \\
Peritumoral LVD & $14.07 \pm 6.08$ & $15.81 \pm 5.31$ & 0.271 \\
\hline
\end{tabular}

Values are presented as mean \pm standard deviation. Group 1: non lymph node metastasis group, Group 2: Iymph node metastasis group.

LVD, lymphatic vessel density.
4. Lymphatic vessel density vs. oral squamous cell carcinoma progression or differentiation

To assess the association between LVD and OSCC progression, the tumors were divided into two groups by primary tumor size at $4 \mathrm{~cm}$. LVD was not associated with tumor size or nodal status (Table 3 ).

5. Lymphatic vessel dilation vs. oral squamous cell carcinoma progression or differentiation

Table 4 shows that higher peritumoral lymphatic vessel dilation values were associated with significantly high scores of N-classification $(P<0.05)$. Moreover, in peritumoral lymphatics, higher lymphatic vessel dilation values were also associated with significantly high proportions of poorly differentiated tumors (Table 5).

\section{Matrix metallproteinases expression in oral squamous} cell carcinoma

We immunostained the specimens with four tissue collagenases (MMP-1, MMP-2, MMP-9, MMP-10) to evaluate their effect on the processes of OSCC progression. MMP-1 and MMP-10 were up-regulated in the stromal area of OSCC. At higher magnification $(\times 1,000)$, the basement membrane of tumor-associated endothelial cells was destroyed by MMP-1 and MMP-10 (Fig. 4).

Table 4. Lymphatic vessel dilation depending on nodal status

\begin{tabular}{lccc}
\hline & Group $1(\mathrm{n}=28)$ & Group $2(\mathrm{n}=16)$ & $P$-value \\
\hline Peritumoral & $0.536 \pm 0.922$ & $1.313 \pm 1.493$ & $0.034^{*}$ \\
Intratumoral & $1.071 \pm 2.071$ & $1.000 \pm 1.366$ & 0.412 \\
\hline
\end{tabular}

Values are presented as mean \pm standard deviation. Group 1: non lymph node metastasis group, Group 2: lymph node metastasis group.

${ }^{*} P<0.05$ (Mann-Whitney test).

Table 5. Lymphatic vessel dilation depending on tumor differentiation

\begin{tabular}{lccc}
\hline & $\begin{array}{c}\text { Well } \\
(n=14)\end{array}$ & $\begin{array}{c}\text { Moderately } \\
(n=9)\end{array}$ & $\begin{array}{c}\text { Poorly } \\
(n=11)\end{array}$ \\
\hline Peritumoral & $0.464 \pm 0.881$ & $0.500 \pm 0.926$ & $2.375 \pm 1.302^{*}$ \\
Intratumoral & $0.714 \pm 1.117$ & $2.250 \pm 3.327$ & $1.000 \pm 1.604$ \\
\hline
\end{tabular}

Values are presented as mean \pm standard deviation. Well: well differentiated SCC, Moderately: moderate differentiated SCC, Poorly: poorly differentiated SCC.

SCC, squamous cell carcinoma.

$* P<0.05$ compared with Moderately and Well (Kruskal-Wallis test). 


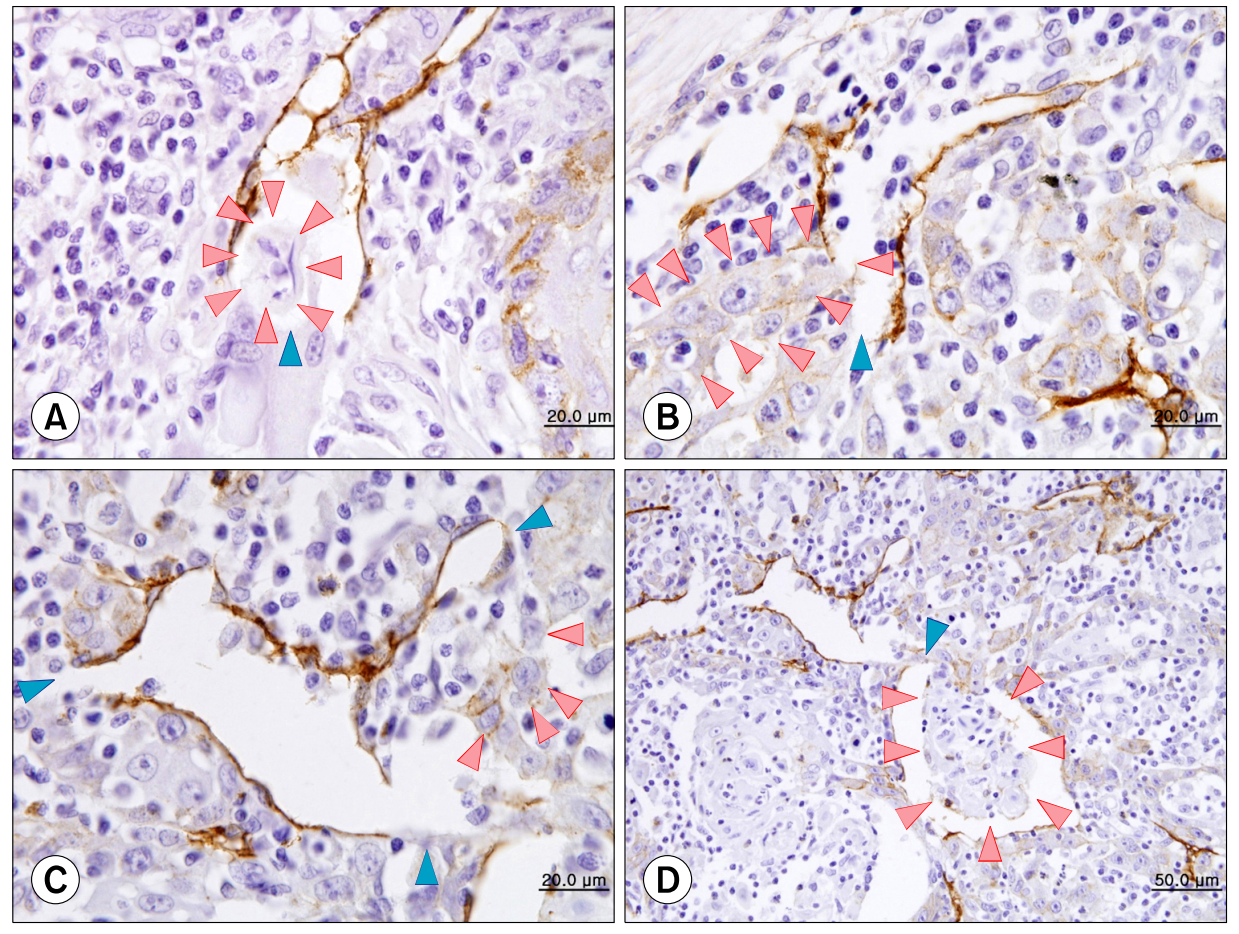

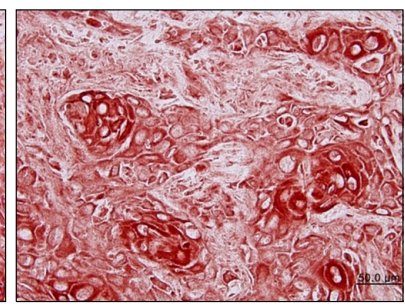
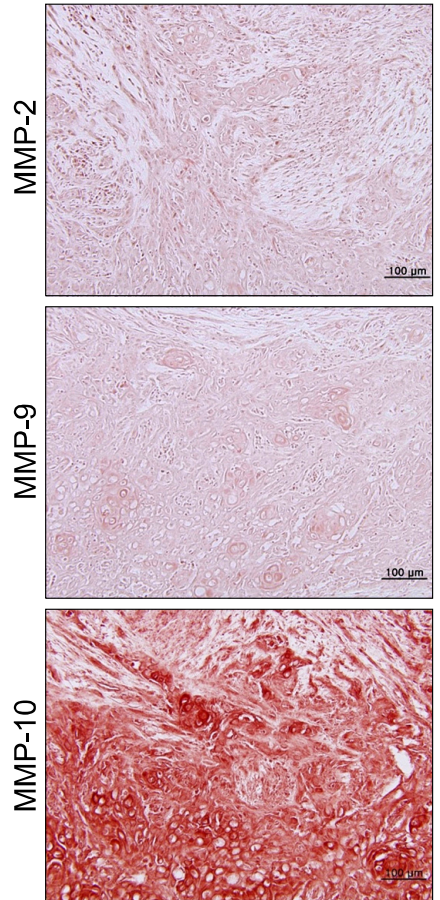
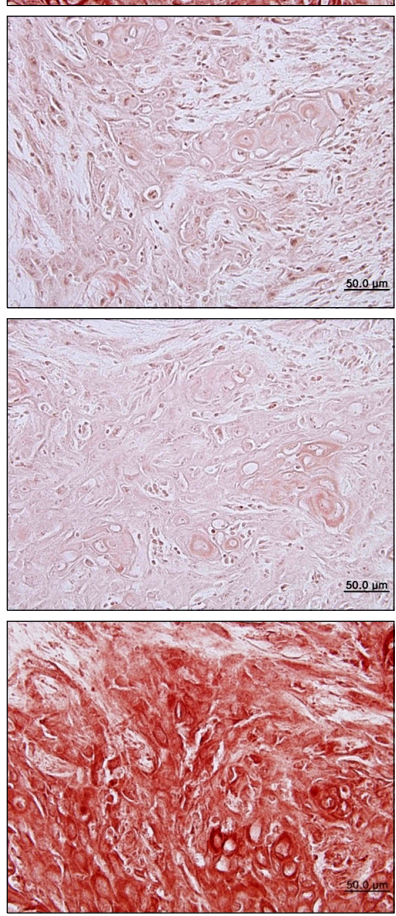
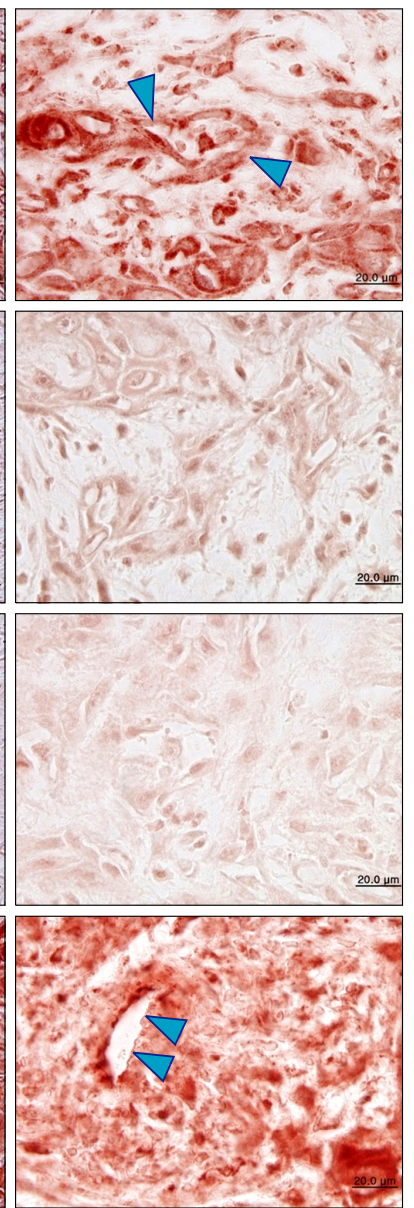

Fig. 3. Invasion of tumor cells (red arrowheads; A C) and a tumor cluster (D) into the ruptured site (blue arrowheads) of dilated lymphatic vessels (A $\sim \mathrm{C}: \times 1,000, \mathrm{D}: \times 400$ ) (counterstained by Mayer's hematoxyline).
Fig. 4. Matrix metalloproteinases (MMPs) expression in oral squamous cell carcinoma; MMP-1 and MMP-10 are overexpressed inoral squamous cell carcinoma. At higher magnification $(\times 1,000)$, the basement membrane of tumor-associated endothelial cells is destroyed by MMP-1 and MMP-10 (blue arrowheads). 


\section{Discussion}

Oral squamous cell carcinoma preferentially metastasizes to cervical lymph nodes, but the cause is unclear. We examined 34 OSCCs on immunohistochemistry using antibodies for lymphangiogenic factors. We found a significant association of increased lymphatic vessel dilatation with nodal metastasis in patients with OSCC. For nodal metastasis, tumor cells must enter the lymphatic system. However, in OSCC as well as other malignancies, it is uncertain whether this is achieved by tumor cells invading the newly formed lymphatic vessels, or by tumor cells entering the expanded preexisting lymphatic vessels near the tumor. Our study shows OSCC tumor cells enter into the dilated lymphatics, newly formed by lymphangiogenesis, to metastasize to the lymph nodes.

OSCC is characterized by aggressive biological behavior with a low five-year survival rate, mainly due to cervical node metastasis[12]. Lymphangiogenic factors are related to cervical nodal metastasis and VEGFR-C is a main stimulator of lymphangiogenesis[13-15]. Tsirlis et al.[16] reported that circulating levels of VEGF-C and VEGF-D serve as predictive biomarkers of lymph node metastasis in gastrointestinal malignancies. Our previous study demonstrated an up-regulation of VEGF-C and VEGF-D in OSCC compared with normal oral mucosa[17]. In the present study, lymphangiogenic ligands are expressed heterogeneously in the tumor cells of OSCC, but their degree of expression is not related with tumor size or nodal metastasis

VEGFR-3 is expressed in the endothelial cells of lymphatic vessels, and the VEGF-C/VEGFR-3 paracrine interaction between tumor cells and lymphatic endothelial cells is the most important system for tumor lymphangiogenesis. VEGFR-3 is also expressed not only in endothelial cells but in OSCC tumor cells[18], and VEGFR-3 expression in tumor cells can be a potential enhancer system to promote cancer progression[19]. Our results also demonstrated that VEGFR-3 is expressed and activated in some tumor cells, suggesting VEGF-C driven autocrine growth loop of VEGF/VEGFR-3 in OSCC.

Frequently, VEGF-C/VEGFR-3 expressions are associated with regional node metastasis and poor patient survival[20-22], but we could not find a relation of VEGF-C,
D/VEGFR-3 expressions to clinicopathologic parameters. The difference between our results and other reports may result from the small sample size of our study. In this study, ten SCC were located in the buccal mucosa, eight in tongue, five in the floor of mouth, three in palate, and so on. Clinically, the predilection of cervical node metastasis is quite different between tongue/mouth floor SCC and palatal/buccal mucosal SCC. The variable location of primary tumors of this study led to small numbers of homogenous samples,

D2-40 positive structures were present in all OSCCs in our study. We found a so-called 'hot spot' of lymphatic vessels, located at the periphery of tumor islands. The area was usually involved in stromal tissue, but morphologically the lymphatic vessels seemed to be tumor-associated, newly formed lymphatics, not preexisting ones. We also observed that in most OSCCs, the hot spot of lymphatic vessels was located in other areas compared to the hot spot of microvessels. Hadler-Olsen et al.[23] reported peritumoral LVD was up to five times higher in tongue SCC than in skin SCC in xenograft murine model and higher in tongue SCCs compared with skin SCCs in a series of patient samples. Our analysis revealed that peritumoral LVD was about two times higher than intratumoral LVD in OSCC. Morphologically, intratumoral lymphatics are compressed, seemingly nonfunctional.

Podoplanin (D2-40) expression in OSCC may be related to its progression[24,25], but our previous study demonstrated that the number of microvessels rather than podoplanin expression was indicative of OSCC progression[26]. In the present study, LVD evaluation revealed that peritumoral LVD and intratumoral LVD are not significantly associated with tumor size or nodal metastasis. Peritumoral lymphatics are dilated in tumors with nodal metastasis or poorly differentiated SCC. Our finding suggests that the number of functional lymphatics are important in OSCC progression.

Sedivy et al.[27] reported that LVD is positively associated with VEGF-C expression and nodal metastasis in OSCC, but we could not reach such a conclusion. This is partly because the difference in determination method of LVD, i.e., we only count the lymphatics with clear lumina. We think morphological changes of newly-formed lymphatics, reflecting functional changes, are important in 
the progression of tumor cells. The cut surface of the dilated lymphatics are polygonal or irregular in shape rather than round or ovoid. The lumina of functional lymphatics are enlarged and the lining endothelium is ruptured to transport tumor cells. In this study, we clearly showed the lymphatic dilation, the ruptured site of lymphatics, and their relation with nodal metastasis.

Tumor progression is strongly influenced by the tumor microenvironment, i.e., tumor stroma, composed of an extracellular matrix, endothelial cells, immune and inflammatory cells. The MMPs are involved in the degradation of the extracellular matrix, and are associated with an aggressive malignant phenotype[28,29]. We immunostained four MMPs on OSCC specimens and found that MMP-1 and MMP-10 are important in the lysis of the glycocalyx inside the tumor-associated lymphatic endothelial cells.

Our findings suggest that in OSCC, nodal metastasis may be achieved mainly through the dilated lymphatic vessels in the periphery of tumor islands. The detection of lymphatic invasion of tumor cells on D2-40 immunostaining in tumor tissue is a strong predictor for nodal metastasis in OSCC. We regard our results as preliminary. The limits of this study include small sample size, the quality of the archival tissue samples, and the sensitivity of antibodies. We need more controlled further study.

\section{Conclusion}

In conclusion, this study indicates that increased dilation of tumor-associated lymphatic vessels is significantly related to nodal metastasis. Furthermore, OSCC tumor cells invaded the ruptured site of the dilated lymphatics, apparently mediated by MMP-1 and MMP-10. In sum, peritumoral lymphatic dilatation may be an important step in the cervical nodal metastasis of oral squamous cell carcinoma. We suggest that newly formed lymphatics inside the tumor are a target for anti-metastasis therapy of OSCC.

\section{Acknowledgements}

This study was supported by a grant of the Korea Healthcare Technology R\&D Project, Ministry of Health \&
Welfare, Republic of Korea (A080293).

We cordially thank to Prof. Suk-Keun Lee at the Department of Oral Pathology, Gangneung-Wonju National University Dental Hospital for his expert interpretation of the histologic data.

\section{References}

1. Fronie A, Bunget A, Afrem E, et al. Squamous cell carcinoma of the oral cavity: clinical and pathological aspects. Rom J Morphol Embryol 2013;54:343-8.

2. Pepper MS. Lymphangiogenesis and tumor metastasis: myth or reality? Clin Cancer Res 2001;7:462-8.

3. Achen MG, Stacker SA. Tumor lymphangiogenesis and metastatic spread-new players begin to emerge. Int J Cancer 2006; 119:1755-60.

4. Wigle JT, Harvey N, Detmar M, et al. An essential role for Prox1 in the induction of the lymphatic endothelial cell phenotype. EMBO J 2002;21:1505-13.

5. Jackson DG, Prevo R, Clasper S, Banerji S. LYVE-1, the lymphatic system and tumor lymphangiogenesis. Trends Immunol 2001;22:317-21.

6. Wicki A, Christofori G. The potential role of podoplanin in tumour invasion. Br J Cancer 2007;96:1-5.

7. Joukov V, Pajusola K, Kaipainen A, et al. A novel vascular endothelial growth factor, VEGF-C, is a ligand for the Flt4 (VEGFR-3) and KDR (VEGFR-2) receptor tyrosine kinases. EMBO J 1996;15:290-8.

8. Witte D, Thomas A, Ali N, Carlson N, Younes M. Expression of the vascular endothelial growth factor receptor-3 (VEGFR-3) and its ligand VEGF-C in human colorectal adenocarcinoma. Anticancer Res 2002;22:1463-6.

9. Brychtova S, Bezdekova M, Brychta T, Tichy M. The role of vascular endothelial growth factors and their receptors in malignant melanomas. Neoplasma 2008;55:273-9.

10. Kaipainen A, Korhonen J, Mustonen T, et al. Expression of the fms-like tyrosine kinase 4 gene becomes restricted to lymphatic endothelium during development. Proc Natl Acad Sci U S A 1995;92:3566-70.

11. O-charoenrat P, Rhys-Evans P, Eccles SA. Expression of vascular endothelial growth factor family members in head and neck squamous cell carcinoma correlates with lymph node metastasis. Cancer 2001;92:556-68.

12. Shah JP, Candela FC, Poddar AK. The patterns of cervical lymph node metastases from squamous carcinoma of the oral cavity. Cancer 1990;66:109-13.

13. Miyahara M, Tanuma J, Sugihara K, Semba I. Tumor lymphangiogenesis correlates with lymph node metastasis and clinicopathologic parameters in oral squamous cell carcinoma. Cancer 2007;110:1287-94.

14. Park YW, Kim SG, Kim SH, Kim HS, Kim MK. Gene expression for lymphangiogenic factors in oral mucosal squamous cell carcinoma. J Korean Assoc Maxillofac Plast Reconstr Surg 2009;31:453-60.

15. Zhang Z, Pan J, Li L, Wang Z, Xiao W, Li N. Survey of risk factors contributed to lymphatic metastasis in patients with or- 
al tongue cancer by immunohistochemistry. J Oral Pathol Med 2011;40:127-34.

16. Tsirlis TD, Papastratis G, Masselou K, et al. Circulating lymphangiogenic growth factors in gastrointestinal solid tumors, could they be of any clinical significance? World J Gastroenterol 2008;14:2691-701.

17. Park YW, Kwon KJ, Lee JW. Immunohistochemical study on expression of lymphangiogenic factors in oral cancer. J Korean Assoc Maxillofac Plast Reconstr Surg 2010;32:1-8.

18. Warburton G, Nikitakis NG, Roberson P, et al. Histopathological and lymphangiogenic parameters in relation to lymph node metastasis in early stage oral squamous cell carcinoma. J Oral Maxillofac Surg 2007;65:475-84.

19. Matsuura M, Onimaru M, Yonemitsu Y, et al. Autocrine loop between vascular endothelial growth factor (VEGF)-C and VEGF receptor-3 positively regulates tumor-associated lymphangiogenesis in oral squamoid cancer cells. Am J Pathol 2009;175:1709-21.

20. Kitadai Y, Amioka T, Haruma K, et al. Clinicopathological significance of vascular endothelial growth factor (VEGF)-C in human esophageal squamous cell carcinomas. Int J Cancer 2001;93:662-6.

21. Tang RF, Itakura J, Aikawa T, et al. Overexpression of lymphangiogenic growth factor VEGF-C in human pancreatic cancer. Pancreas 2001;22:285-92.

22. Amioka T, Kitadai Y, Tanaka S, et al. Vascular endothelial growth factor-C expression predicts lymph node metastasis of human gastric carcinomas invading the submucosa. Eur J Cancer 2002;38:1413-9.

23. Hadler-Olsen E, Wetting HL, Rikardsen O, et al. Stromal impact on tumor growth and lymphangiogenesis in human carcinoma xenografts. Virchows Arch 2010;457:677-92.

24. Funayama A, Cheng J, Maruyama S, et al. Enhanced expression of podoplanin in oral carcinomas in situ and squamous cell carcinomas. Pathobiology 2011;78:171-80.

25. Huber GF, Fritzsche FR, Züllig L, et al. Podoplanin expression correlates with sentinel lymph node metastasis in early squamous cell carcinomas of the oral cavity and oropharynx. Int J Cancer 2011;129:1404-9.

26. Lee SW, Park YW. Expression of endoglin and podoplanin in early and advanced oral squamous cell carcinoma. J Korean Assoc Oral Maxillofac Surg 2012;38:145-51.

27. Sedivy R, Beck-Mannagetta J, Haverkampf C, Battistutti W, Hönigschnabl S. Expression of vascular endothelial growth factor-C correlates with the lymphatic microvessel density and the nodal status in oral squamous cell cancer. J Oral Pathol Med 2003;32:455-60.

28. Chambers AF, Matrisian LM. Changing views of the role of matrix metalloproteinases in metastasis. J Natl Cancer Inst 1997;89:1260-70.

29. Kähäri VM, Saarialho-Kere U. Matrix metalloproteinases and their inhibitors in tumour growth and invasion. Ann Med 1999;31:34-45. 